\title{
Beyond renal dialysis and transplantation - implantable artificial kidneys
}

\begin{abstract}
Alebiosu C.O.
Chronic kidney disease constitutes a public health problem with increasing incidence, prevalence, poor outcomes and high cost. The awareness of and screening for chronic kidney disease remains low worldwide. Thus millions of people around the world suffer from kidney diseases, and these patients eventually will require a form of renal replacement therapy. Outcomes include kidney failure and complications of decreased kidney function with attendant cardiovascular diseases. Dialysis and transplantation, while effective, are not optimal therapies for kidney failure. This is a major reason why researchers have been working on better alternatives including growing 'new kidneys' and creating bioartificial kidney (Kidney Project), to treat end stage renal disease. The Kidney Project' is a US national research project with a goal to create a small, surgically implanted, bioartificial kidney to treat end stage renal disease (ESRD).

The research team is headed by Shuvo Roy, PhD, a bioengineer in the University of California, San Francisco (UCSF), and co-directed by William Fissell, MD, at the Vanderbilt University Medical Center. The bioartificial kidney is expected to save lives and reduce drastically budget committed to renal replacement therapy. After more than 20 years of work, the clinical trials are scheduled to hold early 2018. Although kidney transplantation is still one of the best treatment options of ESRD, unfortunately there is shortage of organ donors that prevents transplants from being available to the vast majority of ESRD patients. Unlike transplants, the bioartificial kidney will not require that patients are on immunosuppressive drugs to prevent rejection ${ }^{2}$. It will be powered by the body's own blood pressure, and does not require external tubes or tethers'. Accordingly to the lead researcher, 'The implantable bioartificial kidney builds upon the existing extracorporeal Renal Assist Device (RAD), which is a bioartificial kidney that combines a membrane hemofilter and a bioreactor of

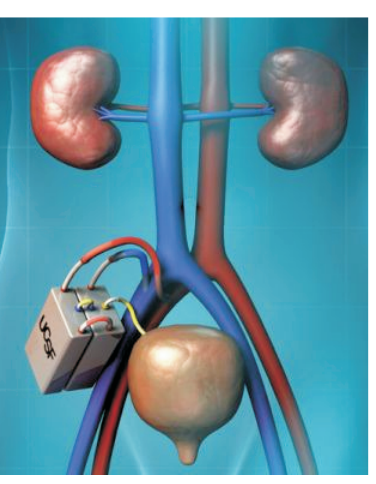
human renal tubule cells to mimic many of the metabolic, endocrine, and immunological functions of a healthy kidney. While clinical trials confirmed that the RAD can safely treat acute renal failure in a critical care setting, adoption of the RAD for routine treatment of ESRD patients is hampered by its labor-intensive and complex operation, large size, and high marginal cost. The ultimate goal of The Kidney Project is to apply micro-electromechanical systems (MEMS) and nanotechnology to miniaturize the extracorporeal RAD into a surgically implantable, self-monitoring, and self-regulating bioartificial kidney.'

In 2014, M. Takasato et al of the University of Queensland's Institute for Molecular Bioscience, grew a primitive kidney from human stem cells ${ }^{3}$. Another group in 2016, grew nephron progenitor cells which differentiate into kidney tissue in vitro ${ }^{4}$. Such research continues, but the ability to grow replacement organs remains a more distant dream than an implantable artificial kidney. It is hoped that in the future, scientists may be able to grow artificial kidneys from body tissues. The Kidney Project is in the process of implanting the bioartificial kidney in human (clinical trial Phase 3). The team anticipates beginning the First-In-Human testing of the hemofilter device by early 2018, assuming no unexpected scientific delays and funding goals are met in a timely manner.
\end{abstract}

\section{REFERENCES}

1.https://pharm.ucsf.edu/kidney

2. https://www.wired.com/story/artificial-kidneys/

3. M. Takasato, P. X. Er, M. Becroft, J. M. Vanslambrouck, E. G. Stanley, A. G. Elefanty et al. Directing human embryonic stem cell differentiation towards a renal lineage generates a self-organizing kidney. Nature Cell Biology 16, 118-126, 2014

4. Cell, Wu et al.: "Interspecies chimerism with mammalian pluripotent stem cells." www.cell.com/cell/fulltext/S00928674(16)31752-4. DOI: $10.1016 /$ j.cell.2016.12.036

Professor Alebiosu C.O.

hettp://orcid.org/0000-0001-6224-6039

Email: olutayo.alebiosu@uniosun.edu.ng

Department of Medicine, College of Health Sciences, Osun State University, Osogbo, Nigeria

Research Journal of Health Sciences subscribed to terms and conditions of Open Access publication. Articles are distributed under the terms of Creative Commons Licence (CC BY-NC-ND 4.0). (http://creativecommons.org/licences/by-nc-nd/4.0). 


\title{
ÉDITORIAL
}

\section{Au-delà de la dialyse rénale et de la transplantation - reins artificiels implantables}

\begin{abstract}
Alebiosu C.O.
La maladie rénale chronique constitue un problème de santé publique avec une incidence croissante, une prévalence, de mauvais résultats et un coût élevé. La sensibilisation et le dépistage de l'insuffisance rénale chronique demeurent faibles partout dans le monde. Ainsi, des millions de personnes à travers le monde souffrent de maladies rénales, et ces patients auront finalement besoin d'une forme de thérapie de remplacement rénal. Les résultats incluent l'insuffisance rénale et les complications de la fonction rénale diminuée avec des maladies cardiovasculaires. La dialyse et la transplantation, bien qu'efficaces, ne sont pas des thérapies optimales pour l'insuffisance rénale. C'est une raison majeure pour laquelle les chercheurs ont travaillé sur de meilleures alternatives, y compris la croissance de «nouveaux reins» et la création de reins bioartificiels (Kidney Project), pour traiter la maladie rénale en phase terminale. The Kidney Project1 est un projet de recherche national américain dont l'objectif est de créer un petit rein bioartificiel implanté chirurgicalement pour traiter l'insuffisance rénale terminale (IRT).

L'équipe de recherche est dirigée par Shuvo Roy, Ph.D., bioingénieur à l'Université de Californie à San Francisco (UCSF) et codirigée par William Fissell, MD, au Vanderbilt University Medical Center. On s'attend à ce que le rein bioartificial sauve des vies et réduise drastiquement le budget engagé à la thérapie de remplacement rénale. Après plus de 20 ans de travail, les essais cliniques devraient avoir lieu début 2018. Bien que la transplantation rénale reste l'une des meilleures options de traitement de l'IRT, malheureusement, il y a pénurie de donneurs d'organes qui empêche la grande majorité des greffes. Patients atteints d'IRT. Contrairement aux transplantations, le rein bioartificiel n'exigera pas que les patients prennent des médicaments immunosuppresseurs pour prévenir le rejet2. Il sera alimenté par la propre pression sanguine du corps et ne nécessite pas de tubes ou de sangles externes2. Selon le chercheur principal, «Le rein bioartificiel implantable s'appuie sur le dispositif d'assistance rénale extracorporelle (RAD), qui est un rein bioartificiel qui combine un hémofiltre membranaire et un bioréacteur de cellules de tubules rénaux humains pour imiter plusieurs des métabolites endocriniens, et les fonctions

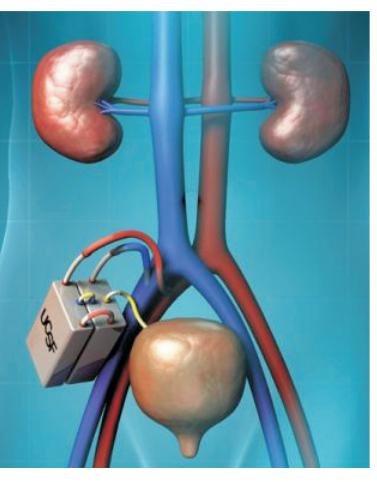
immunologiques d'un rein sain. Alors que les essais cliniques ont confirmé que le RAD peut traiter en toute sécurité l'insuffisance rénale aiguë dans un contexte de soins critiques, l'adoption du RAD pour le traitement de routine des patients IRT est entravée par son fonctionnement complexe, sa grande taille et son coût marginal élevé. Le but ultime de The Kidney Project est d'appliquer les systèmes micro-électromécaniques (MEMS) et la nanotechnologie pour miniaturiser la RAD extracorporelle en un rein bioartificiel chirurgicalement implantable, autosurveillant et autorégulateur.

En 2014, M. Takasato et al., De l'Institute for Molecular Bioscience de l'Université du Queensland, ont cultivé un rein primitif à partir de cellules souches humaines3. Un autre groupe en 2016, a développé des cellules progénitrices néphron qui se différencient en tissu rénal in vitro4. Ces recherches se poursuivent, mais la capacité de développer des organes de remplacement reste un rêve plus lointain qu'un rein artificiel implantable. On espère qu'à l'avenir, les scientifiques pourront produire des reins artificiels à partir de tissus corporels. The Kidney Project est en train d'implanter le rein bioartificiel chez l'humain (essai clinique phase 3). L'équipe prévoit commencer les essais de l'hémofiltre First-In-Human d'ici 2018, en supposant qu'aucun retard scientifique inattendu et aucun objectif de financement ne sont atteints en temps opportun.
\end{abstract}

\section{REFERENCES}

1.https://pharm.ucsf.edu/kidney

2. https://www.wired.com/story/artificial-kidneys/

3. M. Takasato, P. X. Er, M. Becroft, J. M. Vanslambrouck, E. G. Stanley, A. G. Elefanty et al. Directing human embryonic stem cell differentiation towards a renal lineage generates a self-organizing kidney. Nature Cell Biology 16, 118-126, 2014

4. Cell, Wu et al.: "Interspecies chimerism with mammalian pluripotent stem cells." www.cell.com/cell/fulltext/S0092-8674(16)31752-

4. DOI: $10.1016 /$ j.cell.2016.12.036

Professor Alebiosu C.O.

hittp://orcid.org/0000-0001-6224-6039

Email: olutayo.alebiosu@uniosun.edu.ng

Department of Medicine, College of Health Sciences, Osun State University, Osogbo, Nigeria

Research Journal of Health Sciences subscribed to terms and conditions of Open Access publication. Articles are distributed under the terms of Creative Commons Licence (CC BY-NC-ND 4.0). (http://creativecommons.org/licences/by-nc-nd/4.0).

http://dx.doi.org/10.4314/rejhs.v5i4.1 